Conclusion: EC patients with MSI-H status was associated with higher median TMB. TMB varies by underlying cause(s) of MSI, this heterogeneity may contribute to differences in response to ICI.

Poster (E04)

Gestational Trophoblastic Neoplasia

https://doi.org/10.3802/jgo.2021.32.S1.E04

\section{Clinical characteristics and prognostic factors of gestational trophoblastic neoplasia patients: a comparison between ultra high-risk and other risks}

\section{Charuwan Saeteng, " Atita Ruengsaen, Kittipat Charoenkwan, Prapaporn Suprasert, Jatupol Srisomboon \\ Chiang Mai University, Chiang Mai, Thailand (nokctantipa@gmail.com)}

Objective: To evaluate the treatment outcomes and prognostic factors in patients who had ultra high-risk gestational trophoblastic neoplasia (GTN) compared with those who had low-risk and high-risk GTN.

Methods: The medical records of GTN patients treated in Maharaj Nakorn Chiang Mai Hospital between January 1999 and December 2019 were retrospectively reviewed. The overall survival rate was calculated with the Kaplan-Meier method and prognostic factors were analyzed by univariate and multivariate approaches.

Results: During the study period, 160 GTN patients were identified. Of these patients, 98 (61.2\%) were classified as lowrisk, 31 (19.4\%) as high-risk, and 31 (19.4\%) as ultra high-risk GTN. One patient in the low-risk group and 1 in the high -risk group underwent hysterectomy without adjuvant chemotherapy due to spontaneous regression of serum $\beta$-hCG. One patient with ultra high-risk GTN died before receiving chemotherapy. Of 97 low-risk GTN patients, 80 (82.5\%) were treated with either single methotrexate or actinomycin D. Twenty (66.7\%) of 30 high-risk GTN patients were initially treated with EMA/CO (etoposide, methotrexate, actinomycin D, cyclophosphamide, vincristine) regimen as first-line chemotherapy, while 24 (80\%) of 30 patients with ultra high-risk GTN received EMA/CO as first-line chemotherapy. Overall, after completion of treatment with first-line chemotherapy and/or salvage treatment, patients with ultra high-risk GTN had significantly worse treatment outcomes than those who had low- and high-risk GTN with the remission rate of $63.3 \%$ vs. $96.9 \%$ and $80.0 \%$, respectively $(\mathrm{p}<0.01)$. The 5-year overall survival rate of the patients with ultra high-risk GTN was significantly lower than those with low- and high-risk GTN (56\% vs. 96\% and 80\%, respectively, $\mathrm{p}<0.001)$. By multivariable analysis, significant prognostic factors were antecedent term pregnancy with the hazard ratio (HR) of 11.50 (95\% confidence interval $[\mathrm{CI}]=3.56-37.22 ; \mathrm{p}<0.01)$ and brain metastasis $(\mathrm{HR}=4.61 ; 95 \% \mathrm{CI}=1.73-12.28$; $\mathrm{p}<0.01)$. Conclusion: Ultra high-risk GTN patients had significantly worse survival outcomes compared with those who had low-risk and high-risk GTN. The significant prognostic factors were antecedent term pregnancy and brain metastasis.

Poster (E05)

Endometrial Hyperplasia, Endometrial Intra-epithelial Neoplasia, and Endometrial Cancer https://doi.org/10.3802/jgo.2021.32.S1.E05

\section{Endometrial cancer presenting with bone metastasis: a case report and review}

\section{A-jaree Senthong, 'Supapan Wittayanuwat}

Surin Hospital, Surin, Thailand (ajaree0348@gmail.com)

Objective: Endometrial cancer with bone metastasis is rare and extremely poor survival outcome. The 1-year overall survival (OS) is $33.8 \%$. Several factors affect the survival including tumor grade, tumor subtype, liver and brain metastasis. In literatures review, multimodality treatment were independent predictors associated with increase OS.

Methods: Review of a case and literatures.

Results: We present the case of a 59-year-old woman who was referred from the orthopedist to the gynecological clinic with right leg pain and pelvic mass for 3 months. She also had scanty vaginal bleeding. Computed tomography scan showed illdefined enhancing lesion of $9.1 \times 10.7 \times 12.2 \mathrm{~cm}$ sized. It involved both ovaries, uterus, right pelvic sidewall, distal right iliopsoas muscle. Bilateral external iliac lymphadenopathy and peritoneal metastasis were identified. She received endometrial biopsy and the result was poorly differentiated endometrioid carcinoma. Bone metastasis was found in SI joint from bone scan. A diagnosis was an advanced stage endometrial cancer with bone metastasis. Multidisciplinary team planed for treatment with multimodality therapy. She received initial treatment with systemic chemotherapy (carboplatin and paclitaxel) for 3 cycles and followed by pelvic radiation therapy. Hysterectomy and bilateral salpingo-oophorectomy were performed after radiation and additional 6-cycle chemotherapy were given postoperatively. She felt free from leg pain and had normal walk. Unfortunately, she returned to have leg pain 5 months after completion of treatment. The patient is regularly followed up with best palliative care. Conclusion: Bone metastasis from endometrial cancer is rare and has poor prognosis. Multidisciplinary team approach is mandatory to perform appropriate treatment to improve clinical outcome and quality of life. 